\title{
Assessing Building Energy Performance via Selection of Representative Simulation Days
}

\author{
Yan Chen ${ }^{1}$, Saptarshi Bhattacharya ${ }^{1}$, Zhihong Pang ${ }^{2}$, \\ Deepak Sivaraman $^{1}$, Sen Huang ${ }^{1}$, Draguna Vrabie ${ }^{1}$ \\ ${ }^{1}$ Pacific Northwest National Laboratory, Richland, USA \\ ${ }^{2}$ The University of Alabama, Tuscaloosa, USA
}

\begin{abstract}
Simulation-based evaluation and analysis of building system control performance and associated annual energy estimations can become both resource and timeintensive for advanced optimization-based control algorithms. Here we devise a data-driven analysis approach to select a representative subset of test days in a test year, such that those representative days capture seasonal weather variability patterns. We then only compute the energy performance for these representative days and use this reduced data set to compute approximate energy performance estimates for the remaining days through standard prediction approaches. This approach enables us to compute approximate annual energy estimations for building control systems with reduced computation requirements and good accuracy. We evaluate the proposed approach for a few building types and under differing climatic conditions.
\end{abstract}

\section{Introduction}

Building simulation is a traditional approach for assessing and quantifying building energy performance. It has been used for evaluating new building technologies (Ma et al. (2012); Rehrl and Horn (2011); Moroan et al. (2010)), better architectural designs (Clarke (2007); Attia et al. (2012)), and more energy efficient code developments (Athalye et al. (2017); Chen et al. (2019)). The evaluation often requires a simulation analysis for a specified period, such as a couple of days, months, or a whole year. Table 1 provides a brief list of publications where simulation has been used for performance evaluation and associated details on the simulation scenarios. In general, the annual simulation-based analysis provides a compelling and representative result for quantifying the savings from new technology or a new energy code. For example, in ANSI/ASHRAE/IES Standard 90.1 ASHRAE (2016) code analysis, the typical meteorological year (TMY) weather data is used to simulate annual performances of 16 commercial prototype building models at 17 climate locations (Thornton et al. (2011); Athalye et al. (2017)).

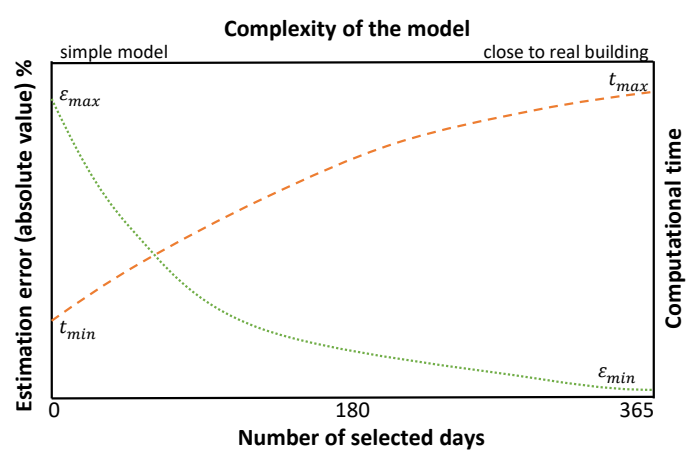

Figure 1: Computation time vs accuracy for performance evaluation.

While annual simulation does provide a comprehensive result, it also requires longer computation time for simulation. The actual computation time can range from less than one minute to several days for a single whole year simulation depending on the complexity and dynamic physical fidelity of the simulation model and the associated simulation solver. Figure 1 provides a graphical description of this observation for computation time and accuracy of performance estimation. A more detailed model yields a more accurate performance estimation result but requires more computation time. Similarly, simulation over a larger number of representative days would provide a more accurate estimate of annual energy usage, and requires larger computation time. To evaluate the performance of advanced control methods in a closed loop with high fidelity simulation models, we devised an approach to predict the annual energy performance for building energy systems by only measuring the control performance for a subset of days, rather than over a year-long simulation.

Several publications have previously presented methods for identifying representative days for demand analysis, instead of using a whole-year demand data. Ortiga et al. (2011) developed a graphic method to select a subset of days for representing the energy demand of the entire year and used TRNSYS as the simulation tool. They showed that the design optimization result that was based on the selected typical days was similar to the result based on the whole year data. 
Table 1: Control studies vary in HVAC system types, location, approach, scenario and evaluation criteria.

\begin{tabular}{|l|l|l|l|l|l|}
\hline Reference & $\begin{array}{l}\text { HVAC system } \\
\text { type }\end{array}$ & Weather location & Testing scenarios & Simulation period & Evaluation metrics \\
\hline Ma et al. (2012) & Single duct multi-zone & Chicago & $\begin{array}{l}\text { Use pre-designed T setpoint } \\
\text { constraints for one day, and } \\
\text { then one week in July }\end{array}$ & 8 days \\
\hline Rehrl and Horn (2011) & Single duct single zone & Austria & $\begin{array}{l}\text { Use step changes of supply air } \\
\text { temperature setpoint as an excitation } \\
\text { to evaluate HVAC system } \\
\text { dynamic response. }\end{array}$ & 1 day \\
\hline Huang (2011) & Single duct single zone & Not specified & $\begin{array}{l}\text { Use step change of the setpoint } \\
\text { as an excitation to evaluate } \\
\text { HVAC system dynamic response. }\end{array}$ & $1-4$ hours \\
\hline Moroan et al. (2010) & $\begin{array}{l}\text { Three zones, individual } \\
\text { zone heating control, } \\
\text { no central AHU. }\end{array}$ & Rennes, France & Use historic weather & 1 day \\
\hline Xi et al. (2007) & Single duct single zone & Not specified & $\begin{array}{l}\text { Use step changes of T setpoint, } \\
\text { and RH\% setpoint. Use different } \\
\text { prediction horizon scenarios. }\end{array}$ & 2 hours \\
\hline Yuan and Perez (2006) & Single duct multi-zone & $\begin{array}{l}\text { Miami, Phoenix, } \\
\text { Nashville, Chicago }\end{array}$ & Use historic weather & $\begin{array}{l}\text { Energy, Comfort, } \\
\text { Computation Time, } \\
\text { Control dynamics }\end{array}$ & Control dynamics \\
\hline Drgoña et al. (2018) & Water-based radiant heating days & Brussels, Belgium & Use historic weather & $\begin{array}{l}\text { Control dynamics, Comfort, } \\
\text { Energy, Indoor Air Quality } \\
\text { (Ventilation) }\end{array}$ \\
\hline
\end{tabular}

Domnguez-Muoz et al. (2011) used a partitional clustering method known as the k-medoids method to select the typical demands days for combined heat and power (CHP) system operation optimization. Similarly, Teichgraebera and Brandta (2018) reviewed the existing papers that focused on clustering analysis and proposed a framework for identifying the typical days for optimization of energy systems.

These references provide methods for selection of typical days for energy demand analysis to optimize the design of the energy system (supply/generation). They focus on categorically reducing the complexity of their experiments through intelligent selection of representative demand days (or profiles), but do not explicitly consider the problem of selecting representative days through consideration of only weather data. Herein we present an approach for selection of a subset of representative days based only on weather data, and we provide a blueprint for estimating annual energy consumption based on the simulation of selected days and data-driven prediction methods. We believe that this approach will guide building operators towards estimating annual energy performance of buildings using minimal computational resources, yet achieving desired estimation accuracy.

The rest of the paper is organized as follows. The Methodology section describes the approach to select the typical days for a given climate location and the approach to estimating annual energy performance. The Test Data Sets section provides a description of the building models and simulation data that we devised for evaluating the approach. The Analysis section presents the results we obtained by applying our proposed approach and its performance when compared with traditional methods based on full-year building energy simulations. The last section concludes this research study and provides an outlook for future research.

\section{Methodology}

Our proposed methodology for the estimation of annual energy consumption includes four major steps (also shown in Figure 2). First, for a given building type, we conduct a correlation analysis to identify the correlation between end-use energy and weather. Energy end-uses that are highly correlated with weather driven load could be predicted based on samples and models that use weather as driving input. Second, we use a clustering method to group different days based on weather feature information. Third, we select/sample days from each cluster and simulate their energy performance. Fourth, we use the energy consumption measured for each of the selected days to derive models of energy consumption that can be used to compute annual energy estimates. Finally, we evaluate the accuracy of the clustering, sampling and estimation methods by comparing the estimated annual energy consumption with the simulated annual energy consumption. The following subsections provide details for each step of this approach.

\section{Step 1: Correlation analysis}

This is the first step for assessing strong influences between weather parameters such as outside air temperature and relative humidity and particular energy end-use in a building system. A significant correlation between a particular energy end-use and the weather parameters provides confidence to predict that enduse by studying the weather parameters exclusively. For example one can compute the Spearman's correlation coefficient (see Myers et al. (2010)) to evaluate the strength of a monotonic relationship between weather features and end-use energy data.

\section{Step 2: Clustering analysis}

Assuming that a particular end-use has appreciable dependence on weather parameters, it is important to group the daily weather patterns based on similarity across multiple weather features. We propose 


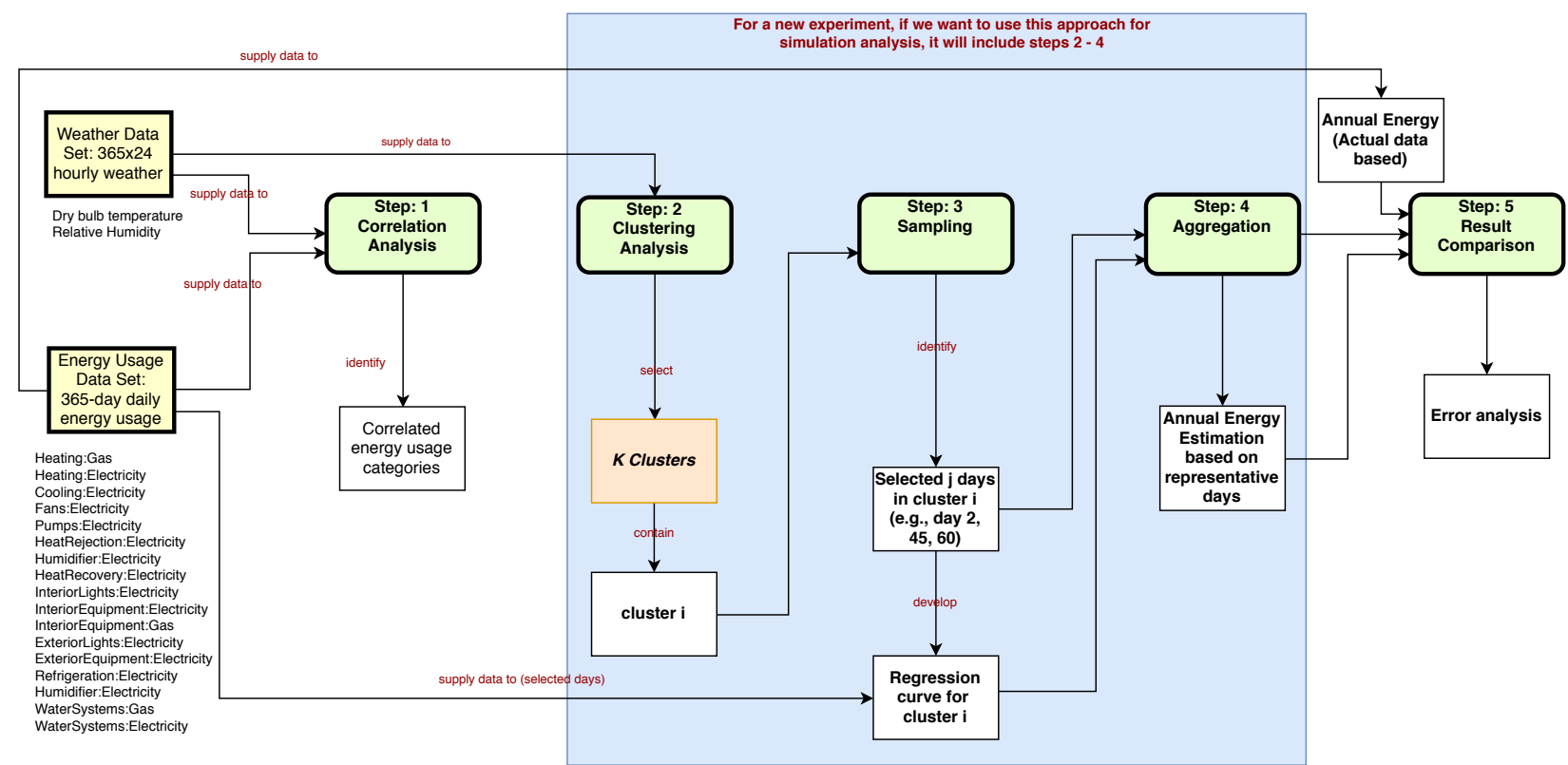

Figure 2: A procedure of estimating annual energy utilization based on selected days.

the utilization of clustering analysis Jain et al. (1999) to capture the relationships between an end-use and weather parameters. We assume that data points within any particular cluster are sufficiently "similar", and an estimate for the end-uses driven substantially by weather variables will be captured with sufficient accuracy by observing a subset of those data points and leveraging existing estimation techniques to approximate the energy associated will other days in the cluster.

Through a clustering technique (e.g., K-means, Kshape or K-medoids), days that have similar weather profile are grouped into similar clusters based on a similarity metric (e.g., the sum of squares driven intra-cluster distance). The "elbow method" (see for example the review paper by Kodinariya and Makwana (2013)) can be used to select the number of clusters based on the intra-cluster variation as a function of the number of clusters. One selects the highest number of clusters after which the intra-cluster variation only improves marginally.

Herein in our numerical analyses we use K-means clustering. Using the original dataset (e.g., the weather data features for the entire year) the algorithm iteratively computes $K$ number of centroids (i.e., means) that minimize the Euclidian distance between data points and selected means. The objective is to minimize intra-cluster (within cluster) variation; as in, to make the dispersion of data points around every centroid as compact as possible.

\section{Step 3: Sampling of Representative Days}

Once the data set is clustered, one needs to select the representative days from each cluster for energy simulation. The simplest approach is to select the points that are closest to the cluster centroids.
However, clustering may result in the formation of clusters which have diverse intra-cluster spread (defined as the maximum distance between two points within a cluster). In other words, some clusters might have a higher scatter of data points, and some clusters may be more compact. When clusters have higher intra-cluster spread, points within that cluster exhibit higher dispersion among their weather attributes compared to a case when a cluster has a lower intra-cluster spread. Therefore any sampling technique which aims to recreate the features of the entire data-set with a high degree of accuracy must consider the differing levels of intra-cluster spreads while deciding the number of points and the approach to sample from each cluster. Assuming that the designer of the experiment has selected the total number of points to be sampled from a full data-set a priori (say this number be $N_{\text {rep }}$ ), we propose a proportional sampling rule, based on the ratio of the intra-cluster spreads of the resulting clusters, where the number of points sampled from a cluster is proportional to its intra-cluster spread.

\section{Step 4: Aggregation}

To compute an approximate of the annual energy consumption, one first runs the energy simulations for the representative days and then uses a methodology to predict the energy consumption for the remaining days. One straightforward approach is to replicate the performance of one representative day in a cluster by the corresponding cluster cardinality to approximate the energy performance for the whole cluster. However, this ignores the variability in weather attributes within a cluster. To address this we propose a method to model and predict the energy associated with the remaining days. 
Let $i$ denote the cluster, $K$ denote the total number of clusters, $d_{j, i}$ denote the day $j$ in cluster $i, D_{i}$ denote the set of all days in cluster $i$, and let $e_{j, i}^{\prime}$ denote the estimated energy usage on day $j$ within cluster $i$. We denote with $E^{\prime}$ the estimated annual energy consumption based on our proposed approach (computing performance for representative days followed by estimation for non-representative days). $w_{j, i}$ denotes the weather data for day $j$ within cluster $i, \mathbf{e}_{s, i}$ denotes the vector of energy consumption for representative days selected from cluster $i$ and $\mathbf{w}_{s, i}$ denotes the vector of weather profiles of representative days chosen from cluster $i$. The total estimated annual energy consumption can be computed as,

$$
E^{\prime}=\sum_{i=1}^{K} \sum_{d_{j, i} \in D_{i}} e_{j, i}^{\prime}
$$

When the aggregation process is purely based on the number of days as a multiplier for the selected days to calculate the energy usage for the whole cluster then

$$
e_{j, i}^{\prime}=e_{s, i}
$$

When a regression model is used to predict the energy usage for each day based on actual weather data then

$$
e_{j, i}^{\prime}=f\left(w_{j, i}, \mathbf{w}_{s, i}, \mathbf{e}_{s, i}\right)
$$

For example, $f$ can be represented as a linear regression model created using the selected days $e_{j, i}^{\prime}=$ $a * w_{i, j}+b$, where $a$ and $b$ represent the regression coefficient generated using the data from selected days $\left(\mathbf{w}_{s, i}, \mathbf{e}_{s, i}\right)$.

\section{Performance evaluation}

To evaluate the accuracy of the estimated annual energy usage $E^{\prime}$ with respect to with the actual annual simulated energy usage $E$, we use

$$
\text { error }=\left(E-E^{\prime}\right) / E \text {. }
$$

Since there are different options in each step from steps 2-4, selection of different options can yield different results. In the following, we select a couple of methods and compare their performances of estimating annual energy usage using our proposed process (see Table 2).

\section{Test Data Sets}

We prepared a few data sets representative for different building types, building vintages, and climate locations. These datasets were generated from the commercial prototype building models developed by building energy codes program U.S. Building Energy Codes Program (2018). The prototype models include 16 different commercial building types

\begin{tabular}{|c|c|c|c|}
\hline No. & Clustering variable & Sampling & Aggregation \\
\hline 1 & $T_{\text {mean }}$ & \multirow{4}{*}{$\begin{array}{l}\text { a day that is the } \\
\text { closest to the centroid } \\
\text { of each cluster }\end{array}$} & \multirow{4}{*}{$\begin{array}{l}\text { direct multiplying } \\
\text { by cluster size }\end{array}$} \\
\hline 2 & $T_{\text {median }}$ & & \\
\hline 3 & $T_{\text {mean }}+S T D_{T}$ & & \\
\hline 4 & $T_{\text {median }}+S T D_{T}$ & & \\
\hline 5 & $T_{\text {mean }}$ & \multirow{2}{*}{$\begin{array}{l}\text { a (number of) } \\
\text { day (s) weighted } \\
\text { based on cluster size }\end{array}$} & $\begin{array}{l}\text { linear regression } \\
\text { based aggregation } \\
\text { using } T_{\text {mean }} \\
\text { for each cluster }\end{array}$ \\
\hline 6 & $T_{\text {mean }}, R H_{\text {mean }}$ & & $\begin{array}{l}\text { linear regression } \\
\text { based aggregation } \\
\text { using } T_{\text {mean }}, R H_{\text {mean }} \\
\text { for each cluster }\end{array}$ \\
\hline
\end{tabular}
in 17 climate locations, representing different energy
Table 2: List of methods for the demonstration case study.

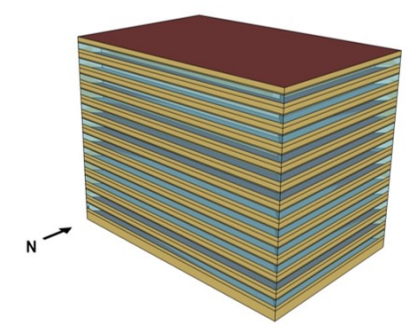

Figure 3: Geometry of the prototype building model: Large Office.

codes requirement (i.e., from ASHRAE 90.1-2004 to ASHRAE 90.1-2016, from IECC 2006 to IECC 2015). These models are developed based on EnergyPlus 8.0. These building models are not calibrated against real building energy usage, and the annual simulation results have been used for quantifying energy saving from building energy codes developments (Athalye et al. (2017); Thornton et al. (2011)) . For this case study, we selected prototype models to include a variety of building type (i.e., large office (Figure 3) and large hotel (Figure 4)), climate location (i.e., Chicago, and San Francisco), and building construction vintage (i.e., complied with 90.1-2013). As a result, we generated four datasets that each represents a 365day daily energy usage profile.

Large office building model represents a 12-floor rectangular box shape building. Each floor is served by a single duct Variable Air Volume (VAV) multizone system. It has a weekly working and occupancy schedule. Large hotel building model represents the lodging type of building and has 6 floors above the ground and one basement. The public spaces are served by a VAV system, while the guest rooms are served by dedicated outside air system and fan-coil units.

We first simulated the building model for the whole

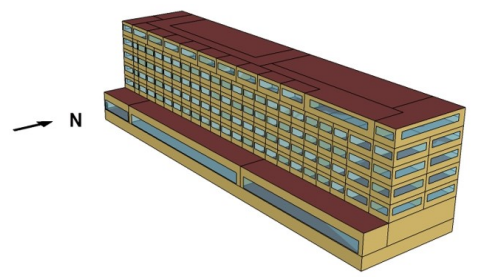

Figure 4: Geometry of the prototype building model: Large Hotel. 


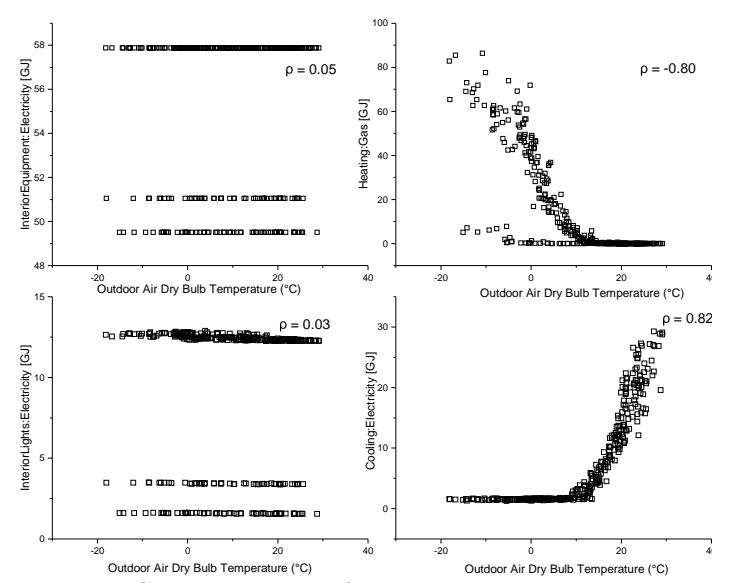

Figure 5: Scatter plot of top-4 energy end-use comparing to outdoor air dry bulb temperature (Large Office in Chicago).
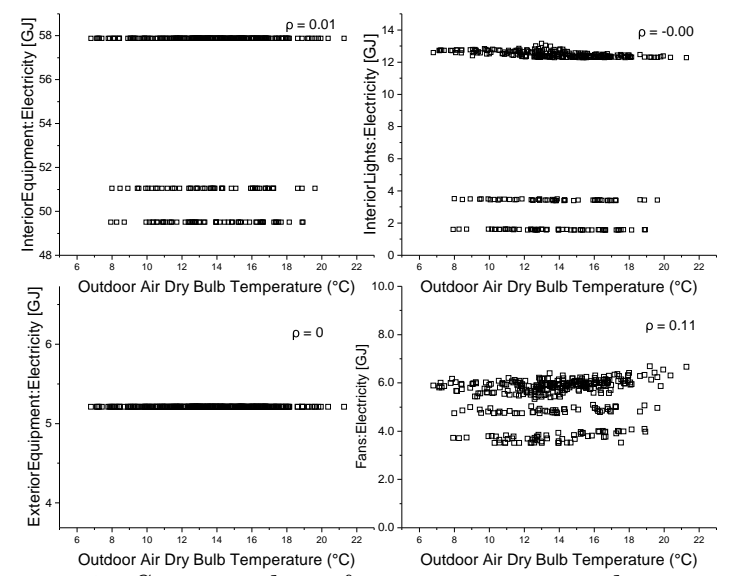

Figure 6: Scatter plot of top-4 energy end-use comparing to outdoor air dry bulb temperature (Large Office in San Francisco).

year period to generate the data as the ground-truth. Please note that the simulation used TMY weather data. Daily energy by energy end-use type is reported as the simulation output. The energy end-use include electrical Heating, cooling, fan, pumps, heat rejection, humidifier, heat recovery, lighting, and plug load.

\section{Analysis}

\section{Correlation analysis}

Using the 365-day daily energy end-use data sets, we present selected scatter plots in Figure 5 to 7 . Each figure shows the top- 4 building energy end-use categories and how they relate to outdoor air dry bulb temperature. Visual inspection suggests that there are different types of correlation between energy enduse and the outdoor air temperature. One can use also compute the correlation coefficients such as Pearson and Spearman to evaluate the statistical dependence between two variables. We make the following observations:

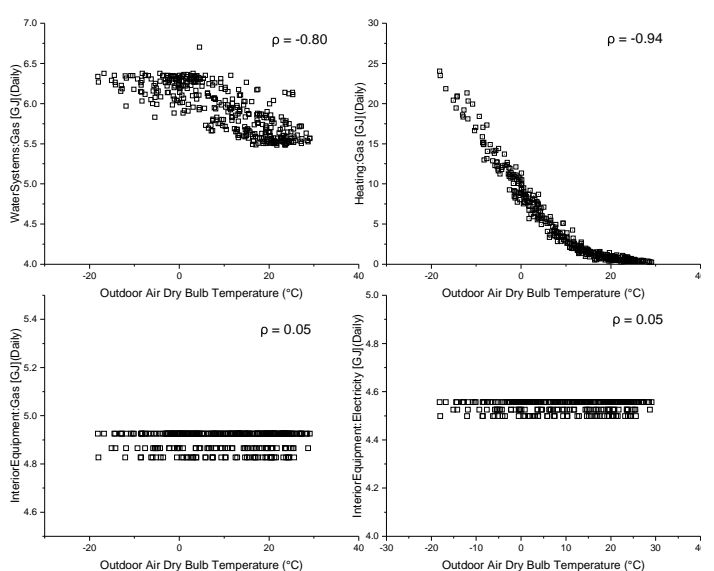

Figure 7: Scatter plot of top-4 energy end-use comparing to outdoor air dry bulb temperature (Large Hotel in Chicago).

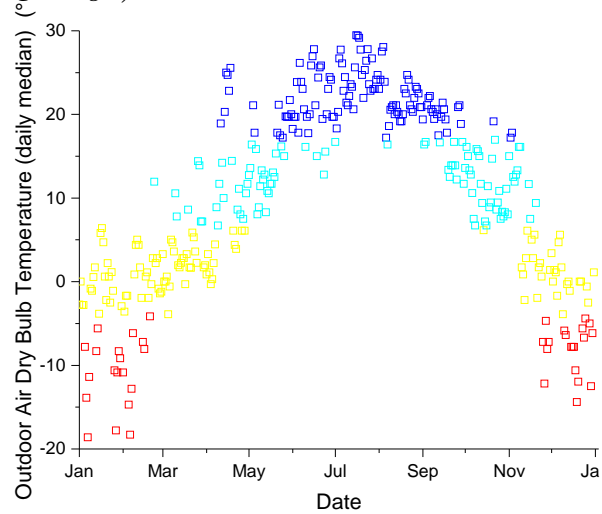

Figure 8: Cluster of daily median dry bulb temperature in Chicago.

1. The ranking of top energy end-use categories is specific to building type and climate location: for example, in Chicago, the top-4 energy end-use types are different between the large office building and the large hotel building;

2. Heating energy and cooling energy is highly correlated to the weather data, while energy usage from lighting and interior equipment are stratified and correlate with daily schedules;

3. For a certain energy end-use category, the shape of the correlation between this type of energy enduse and the weather data remain relatively the same regardless of climate location.

\section{Clustering}

Each method listed in Table 2 generated a clustering result. Here, due to the page limit, we present four clustering results below. Figure 8 shows the Kmeans clustering of weather (dry-bulb temperature) for Chicago, and Figure 9 shows the result for San Francisco. Figure 10 shows the K-mean clustering of weather using two weather parameters (dry bulb temperature and relative humidity $(\mathrm{RH})$ ). As can be seen from the clustering results, individual days represented by the weather information are grouped into different clusters. Each cluster contains the days that are mostly alike with each other. 


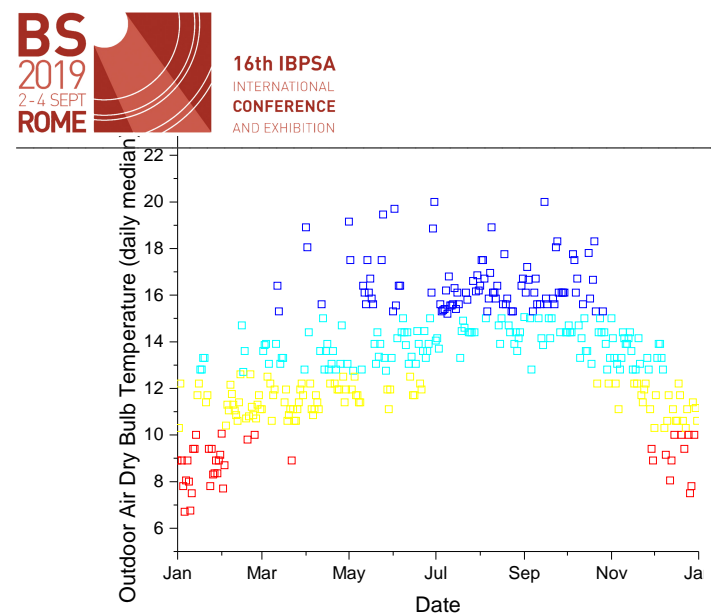

Figure 9: Cluster of daily median dry bulb temperature in San Francisco.

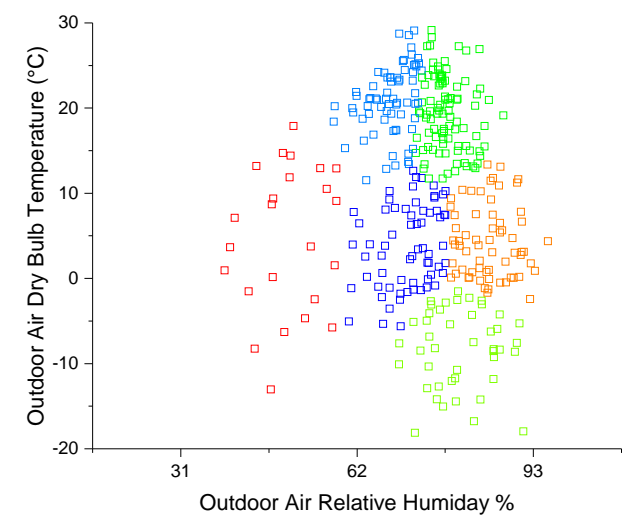

Figure 10: Cluster of daily mean dry bulb temperature and relative humidity in Chicago.

\section{Sampling}

As mentioned in the method section, there are two main options for sampling. Under the first option select one day from each cluster, the day that is the closest to the idea center point is selected (see Table 3 ). Under the second option - select multiple days based on the size of the cluster, at least three days were selected from one cluster, which aims to supply enough data points for the regression process at the "Aggregation" step.Table 3 shows an example of the selected representative days for Chicago under using $T_{\text {mean }}$, and $T_{\text {mean }}, S T D_{T}$. As can be seen, although different days are selected as the representative days for different cases, the selected dates do cover each season (i.e., spring, summer, fall, and winter).

Table 3: An example of sampling result - representative days in each cluster.

\begin{tabular}{|c|c|c|c|c|}
\hline Weather Data & Method & Cluster \# & Selected date & $\begin{array}{r}\text { Number of days } \\
\text { in cluster }\end{array}$ \\
\hline \multirow{20}{*}{ Chicago } & \multirow{4}{*}{$T_{\text {mean }}$} & 1 & 30-Dec & 40 \\
\hline & & 2 & 2-Mar & 99 \\
\hline & & 3 & 16-May & 97 \\
\hline & & 4 & 31-Aug & 129 \\
\hline & \multirow{16}{*}{$T_{\text {mean }}$ and $S T D_{T}$} & 1 & 29-Jan & 11 \\
\hline & & 2 & $26-\mathrm{Nov}$ & 16 \\
\hline & & 3 & 30-Jan & 9 \\
\hline & & 4 & 30-Dec & 4 \\
\hline & & 5 & 24-Mar & 48 \\
\hline & & 6 & 9-Jan & 35 \\
\hline & & 7 & $17-\mathrm{Feb}$ & 14 \\
\hline & & 8 & 18-Mar & 2 \\
\hline & & 9 & 8-May & 25 \\
\hline & & 10 & 23-Oct & 37 \\
\hline & & 11 & 13-Sep & 25 \\
\hline & & 12 & 24-Apr & 10 \\
\hline & & 13 & 3-Nov & 13 \\
\hline & & 14 & 17-Jun & 50 \\
\hline & & 15 & 13-Jul & 53 \\
\hline & & 16 & 22-May & 13 \\
\hline
\end{tabular}

Aggregation (Annual Energy Estimation)

Based on energy data from selected days, the annual energy is calculated using the aggregation method following Eq. 1. The results are presented in the form of error percentage for each end-use in the Table 4 to Table 6 and the total HVAC and non-HVAC summary is presented in Table 7 . When the energy end-use is not weather depended it is sufficient to select one single day from each cluster and use naive estimation to obtain good performance. When the energy end-uses are strongly weather depended, selection of a single day from each cluster is insufficient to accurately approximate annual energy usage. The use of regression-based approximation results in reduced estimation errors. For example, for the large office in Chicago (see Figure 4, linear regression based on selected weather variables resulted in reduced errors in annual energy estimates. However, we also observe some increases of error, possibly due utilization of linear regression on feature variables that have a non-linear correlation with those certain energy enduses.

As expected the use of a larger number of samples will yield an improved estimation performance for annual energy. The regression process captures close relationships between a type of energy end use and the weather conditions leading to significantly improved the estimation accuracy when compared to simple aggregation approaches. The proposed approach can significantly expedite the time to obtain an annual performance estimate associated with a given building system. This method could be further improved in the following aspects:

- explore the use of different clustering methods and different weather features;

- develop aggregation methods that offer performance guarantees;

- explore the use of hourly and weekly sample length data and compare with daily samples.

We are also interested in evaluating the performance of this method applying for other building types and construction vintages.

\section{Conclusion}

Here we presented a sampling-based method to reduce the computation time required for simulationbased annual building energy performance evaluation. Existing approaches make use of whole year simulation data to evaluate the energy performance of different end-uses. Our method provides a constructive approach to select a small set of test days based on the analysis of annual weather data and uses the test results to compute an approximate estimate for the annual energy consumption. The method includes clustering analysis to select representative days, and sampling and regression methods to compute the annual energy estimate. In the Analysis section, we 
Table 4: Estimation error for different energy end-uses (Large Office in Chicago).

\begin{tabular}{|c|c|c|c|c|c|c|c|}
\hline Energy End Use Category & Annual Simulation (GJ) & T mean & T median & T mean +STD $\mathrm{T}$ & $\mathrm{T}$ median + STD $\mathrm{T}$ & LR (T mean) & LR ( $\mathrm{T}$ mean, RH mean) \\
\hline Heating:Gas & 5325.35 & $10 \%$ & $24 \%$ & $13 \%$ & $-3 \%$ & $-10 \%$ & $15 \%$ \\
\hline Heating:Electricity & 10.80 & $24 \%$ & $27 \%$ & $-42 \%$ & $7 \%$ & $-30 \%$ & $10 \%$ \\
\hline Cooling:Electricity & 2748.36 & $23 \%$ & $-30 \%$ & $-3 \%$ & $17 \%$ & $4 \%$ & $-2 \%$ \\
\hline Fans:Electricity & 2192.12 & $7 \%$ & $-4 \%$ & $5 \%$ & $1 \%$ & $4 \%$ & $-1 \%$ \\
\hline Pumps:Electricity & 463.00 & $17 \%$ & $-12 \%$ & $-2 \%$ & $12 \%$ & $1 \%$ & $3 \%$ \\
\hline HeatRejection:Electricity & 260.77 & $29 \%$ & $-44 \%$ & $-8 \%$ & $28 \%$ & $4 \%$ & $-8 \%$ \\
\hline Humidifier:Electricity & 1599.05 & $-3 \%$ & $7 \%$ & $1 \%$ & $3 \%$ & $-4 \%$ & $-2 \%$ \\
\hline HeatRecovery:Electricity & 52.08 & $-23 \%$ & $-100 \%$ & $0 \%$ & $61 \%$ & $40 \%$ & $20 \%$ \\
\hline InteriorLights:Electricity & 3411.15 & $22 \%$ & $-6 \%$ & $10 \%$ & $-4 \%$ & $11 \%$ & $-3 \%$ \\
\hline InteriorEquipment:Electricity & 20256.63 & $3 \%$ & $-1 \%$ & $1 \%$ & $0 \%$ & $2 \%$ & $0 \%$ \\
\hline ExteriorLights:Electricity & 684.28 & $-3 \%$ & $0 \%$ & $-3 \%$ & $-7 \%$ & $-1 \%$ & $0 \%$ \\
\hline ExteriorEquipment:Electricity & 1900.91 & $0 \%$ & $0 \%$ & $0 \%$ & $0 \%$ & $0 \%$ & $0 \%$ \\
\hline WaterSystems:Gas & 573.14 & $19 \%$ & $-2 \%$ & $9 \%$ & $-3 \%$ & $10 \%$ & $-1 \%$ \\
\hline
\end{tabular}

Table 5: Estimation error for different energy end-uses (large Hotel in Chicago).

\begin{tabular}{|c|c|c|c|c|c|c|c|}
\hline Energy End Use Category & Annual Simulation (GJ) & T mean & T median & $\mathrm{T}$ mean + STD $\mathrm{T}$ & T median +STD $\mathrm{T}$ & LR ( T mean) & LR ( T mean, RH mean) \\
\hline Heating:Gas & 1855.97 & $-6.2 \%$ & $-8.5 \%$ & $10.5 \%$ & $-3.2 \%$ & $2.5 \%$ & $0 \%$ \\
\hline Cooling:Electricity & 1106.60 & $-26.1 \%$ & $-6.2 \%$ & $-32.2 \%$ & $8.5 \%$ & $-4.5 \%$ & $0 \%$ \\
\hline Fans:Electricity & 663.91 & $-1.4 \%$ & $0.9 \%$ & $-0.8 \%$ & $-0.2 \%$ & $1.5 \%$ & $-1 \%$ \\
\hline Pumps:Electricity & 71.23 & $-2.8 \%$ & $-5.8 \%$ & $-5.2 \%$ & $-0.6 \%$ & $0.0 \%$ & $-3 \%$ \\
\hline HeatRecovery:Electricity & 179.87 & $7.5 \%$ & $-8.1 \%$ & $-13.6 \%$ & $6.7 \%$ & $13.5 \%$ & $-1 \%$ \\
\hline InteriorLights:Electricity & 914.53 & $0.4 \%$ & $0.5 \%$ & $-0.4 \%$ & $-0.7 \%$ & $0.8 \%$ & $0 \%$ \\
\hline InteriorEquipment:Electricity & 1658.22 & $0.2 \%$ & $0.2 \%$ & $-0.1 \%$ & $-0.2 \%$ & $0.8 \%$ & $0 \%$ \\
\hline InteriorEquipment:Gas & 1788.91 & $0.3 \%$ & $0.4 \%$ & $-0.2 \%$ & $-0.3 \%$ & $0.8 \%$ & $0 \%$ \\
\hline ExteriorLights:Electricity & 234.89 & $-3.5 \%$ & $0.2 \%$ & $-2.6 \%$ & $-6.1 \%$ & $1.5 \%$ & $0 \%$ \\
\hline ExteriorEquipment:Electricity & 950.45 & $0.0 \%$ & $0.0 \%$ & $0.0 \%$ & $0.0 \%$ & $0.8 \%$ & $0 \%$ \\
\hline Refrigeration:Electricity & 61.40 & $2.7 \%$ & $2.5 \%$ & $1.5 \%$ & $-0.2 \%$ & $0.1 \%$ & $1 \%$ \\
\hline WaterSystems:Gas & 2163.16 & $-0.1 \%$ & $0.6 \%$ & $0.1 \%$ & $0.3 \%$ & $1.6 \%$ & $0 \%$ \\
\hline WaterSystems:Electricity & 90.74 & $-0.4 \%$ & $-0.5 \%$ & $0.2 \%$ & $0.4 \%$ & $0.9 \%$ & $1 \%$ \\
\hline
\end{tabular}

Table 6: Estimation error for different energy end-uses (Large Office in San Francisco).

\begin{tabular}{|c|c|c|c|c|c|c|c|}
\hline Energy End Use Category & Annual Simulation (GJ) & T mean & T median & T mean + STD $\mathrm{T}$ & T median +STD T & LR (T mean) & LR (T mean, RH mean) \\
\hline Heating:Gas & 172.29 & $-20 \%$ & $33 \%$ & $-20 \%$ & $79 \%$ & $2 \%$ & $-30 \%$ \\
\hline Heating:Electricity & 10.26 & $-69 \%$ & $-59 \%$ & $-22 \%$ & $-11 \%$ & $9 \%$ & $37 \%$ \\
\hline Cooling:Electricity & 1352.62 & $-10 \%$ & $-18 \%$ & $-1 \%$ & $0 \%$ & $-3 \%$ & $-7 \%$ \\
\hline Fans:Electricity & 1969.80 & $0 \%$ & $5 \%$ & $3 \%$ & $-1 \%$ & $-5 \%$ & $2 \%$ \\
\hline Pumps:Electricity & 262.09 & $-12 \%$ & $-5 \%$ & $0 \%$ & $-4 \%$ & $-3 \%$ & $-7 \%$ \\
\hline HeatRejection:Electricity & 99.34 & $-22 \%$ & $-32 \%$ & $-20 \%$ & $17 \%$ & $-18 \%$ & $-14 \%$ \\
\hline Humidifier:Electricity & 509.21 & $-60 \%$ & $-50 \%$ & $-5 \%$ & $111 \%$ & $33 \%$ & $-5 \%$ \\
\hline InteriorLights:Electricity & 3417.02 & $-4 \%$ & $35 \%$ & $15 \%$ & $-3 \%$ & $-15 \%$ & $15 \%$ \\
\hline InteriorEquipment:Electricity & 20256.63 & $0 \%$ & $4 \%$ & $2 \%$ & $0 \%$ & $-2 \%$ & $2 \%$ \\
\hline ExteriorLights:Electricity & 680.09 & $0 \%$ & $6 \%$ & $-6 \%$ & $9 \%$ & $0 \%$ & $-1 \%$ \\
\hline ExteriorEquipment:Electricity & 1900.91 & $0 \%$ & $0 \%$ & $0 \%$ & $0 \%$ & $0 \%$ & $0 \%$ \\
\hline WaterSystems:Gas & 532.45 & $2 \%$ & $29 \%$ & $11 \%$ & $-4 \%$ & $-12 \%$ & $11 \%$ \\
\hline
\end{tabular}

Table 7: Summary of the estimation error for different scenarios.

\begin{tabular}{|r|r|r|r|r|r|r|r|r|}
\hline $\begin{array}{r}\text { Building Type } \\
\text { and Location }\end{array}$ & $\begin{array}{r}\text { Energy Usage } \\
\text { Cat. }\end{array}$ & $\begin{array}{r}\text { Ground } \\
\text { Truth (GJ) }\end{array}$ & T mean & T median & $\begin{array}{r}\text { T mean } \\
\text { +STD T }\end{array}$ & $\begin{array}{r}\text { T median } \\
\text { +STD T }\end{array}$ & $\begin{array}{r}\text { LR } \\
\text { (T mean) }\end{array}$ & $\begin{array}{r}\text { LR mean, } \\
\text { RH mean) }\end{array}$ \\
\hline \multirow{2}{*}{$\begin{array}{r}\text { Large Office, } \\
\text { Chicago }\end{array}$} & HVAC & 12652 & $11.0 \%$ & $1.8 \%$ & $5.2 \%$ & $4.1 \%$ & $-3.0 \%$ & $5.4 \%$ \\
\cline { 2 - 9 } & Other & 26826 & $5.3 \%$ & $-1.4 \%$ & $2.3 \%$ & $-1.2 \%$ & $2.8 \%$ & $-0.6 \%$ \\
\hline \multirow{2}{*}{\begin{tabular}{r} 
Large Hotel, \\
\cline { 2 - 9 } Chicago
\end{tabular}} & Total & 39478 & $7.2 \%$ & $-0.4 \%$ & $3.3 \%$ & $0.5 \%$ & $0.9 \%$ & $1.3 \%$ \\
\cline { 2 - 9 } & HVAC & 3878 & $-10.3 \%$ & $-6.2 \%$ & $-5.0 \%$ & $1.2 \%$ & $0.8 \%$ & $-0.3 \%$ \\
\hline \multirow{2}{*}{$\begin{array}{r}\text { Large Office, } \\
\text { San Francisco }\end{array}$} & Other & 7862 & $0.1 \%$ & $0.4 \%$ & $-0.1 \%$ & $-0.3 \%$ & $1.1 \%$ & $0.2 \%$ \\
\cline { 2 - 9 } & Total & 11740 & $-3.4 \%$ & $-1.8 \%$ & $-1.8 \%$ & $0.2 \%$ & $1.0 \%$ & $0.0 \%$ \\
\cline { 2 - 9 } & HVAC & 4376 & $-12.4 \%$ & $-9.0 \%$ & $-0.9 \%$ & $15.8 \%$ & $0.6 \%$ & $-3.7 \%$ \\
\hline
\end{tabular}


presented results that demonstrate that the approach can lead to $<5 \%$ annual estimates for most end uses. Further research will be focused on the development of sampling and annual estimation methods which enhance the prediction accuracy and provide specific performance bounds. A specific use case for this approach will be an evaluation of the annual performance of advanced and computationally intensive control algorithms such as model-based predictive control.

\section{Acknowledgment}

This work has been supported by the Building Technologies Office of the U.S. Department of Energy's Office of Energy Efficiency and Renewable Energy.

\section{References}

ASHRAE (2016). Ansi/ashrae/ies 90.1-2016, energy standard for buildings except low-rise residential buildings.

Athalye, R., M. Halverson, M. Rosenberg, B. Liu, J. Zhang, R. Hart, V. Mendon, S. Goel, Y. Chen, Y. Xie, and M. Zhao (2017). Energy savings analysis: Ansi/ashrae/ies standard 90.1-2016.

Athalye, R. A., Y. Chen, J. Zhang, B. Liu, M. Frankel, and M. Lyles (2017). City reach code technical support document.

Attia, S., E. Gratia, A. De Herde, and J. L. Hensen (2012). Simulation-based decision support tool for early stages of zero-energy building design. Energy and buildings 49, 2-15.

Chen, Y., B. Liu, J. Zhang, M. I. Rosenberg, J. Edelson, and M. Lyles (2019). Final energy savings analysis of the proposed nystretch-energy code 2018.

Clarke, J. (2007). Energy simulation in building design. Routledge.

Domnguez-Muoz, F., J. M. Cejudo-Lpez, A. CarrilloAndrs, and M. Gallardo-Salazar (2011). Selection of typical demand days for chp optimization. Energy and Buildings 43(11), 3036 - 3043.

Drgoňa, J., D. Picard, M. Kvasnica, and L. Helsen (2018). Approximate model predictive building control via machine learning. Applied Energy 218, 199-216.

Huang, G. (2011). Model predictive control of vav zone thermal systems concerning bi-linearity and gain nonlinearity. Control Engineering Practice 19(7), 700-710.

Jain, A. K., M. N. Murty, and P. J. Flynn (1999). Data clustering: a review. ACM computing surveys (CSUR) 31(3), 264-323.
Kodinariya, T. M. and P. R. Makwana (2013). Review on determining number of cluster in kmeans clustering. International Journal of Advance Research in Computer Science and Management Studies 1(6), 90-95.

Ma, J., J. Qin, T. Salsbury, and P. Xu (2012). Demand reduction in building energy systems based on economic model predictive control. Chemical Engineering Science 67(1), 92-100.

Moroan, P.-D., R. Bourdais, D. Dumur, and J. Buisson (2010). Building temperature regulation using a distributed model predictive control. Energy and Buildings 42(9), 1445-1452.

Myers, J., A. Well, and R. Lorch (2010). Research Design and Statistical Analysis. Research Design and Statistical Analysis. Routledge.

Ortiga, J., J. Bruno, and A. Coronas (2011). Selection of typical days for the characterisation of energy demand in cogeneration and trigeneration optimisation models for buildings. Energy Conversion and Management 52(4), 1934 - 1942.

Rehrl, J. and M. Horn (2011). Temperature control for hvac systems based on exact linearization and model predictive control. In IEEE International Conference on Control Applications (CCA), pp. 1119-1124.

Teichgraebera, H. and A. R. Brandta (2018). Clustering methods to find representative periods for the optimization of energy systems: an initial framework and comparison. In Preprint submitted to optimization-online.org.

Thornton, B. A., M. I. Rosenberg, E. E. Richman, W. Wang, Y. Xie, J. Zhang, H. Cho, V. V. Mendon, R. A. Athalye, and B. Liu (2011). Achieving the $30 \%$ goal: Energy and cost savings analysis of ashrae standard 90.1-2010.

U.S. Building Energy Codes Program (2018). Commercial prototype building models. https://www . energycodes.gov/development/ commercial/prototype_models Accessed: 201901-02.

Xi, X.-C., A.-N. Poo, and S.-K. Chou (2007). Support vector regression model predictive control on a hvac plant. Control Engineering Practice 15(8), 897908.

Yuan, S. and R. Perez (2006). Multiple-zone ventilation and temperature control of a single-duct vav system using model predictive strategy. Energy and Buildings 38(10), 1248-1261. 